\title{
New Dynamic Battery Model for Hybrid Vehicles and Dynamic Model Analysis Using Simulink.
}

\section{S. M. Wijewardana}

\begin{abstract}
Mathematical modeling and dynamic simulation of battery storage systems can be challenging and demanding due to nonlinear nature. Simulation in time domain could be time consuming as battery storage (BS) systems do not behave according to readily available mathematical functions. Economic advantages, partial sustainability and the portability of these units pose promising substitutes for backup power systems in hybrid vehicles, hybrid electricity power generation systems, telecommunication exchanges and computer networks. Though, there have been many research papers published in this area with complex mathematical models and simulation systems, each system has its own constraints and specific applications. The aim of this research paper is to present a suitable convenient, dynamic battery model that can be used to model a general BS system. The proposed new dynamic battery model has the capability to analyse the effect of temperature, cyclic charging/discharging, and voltage stabilization effects. Simulink has been used to study the characteristics of the system and the proposed system has produced very good successful results.
\end{abstract}

Keywords: Simulink/Matlab, Battery Model, Dynamic modeling, Hybrid Vehicles, Simulation.

\section{Introduction}

Energy storage systems are crucial for the future Smart Grid and Hybrid Vehicles. Their ability to store energy has changed the traditional power system's definition. However, the benefits are hard to quantify because of the complexity of different applications and modeling difficulties. Dynamic modeling has become even harder to count all aspects of battery life cycle, selfdischarging, gassing effect, diffusion process, acid stratification, state of charge etc[1]. Analysing various battery models presented by previous researchers, it was found that there is a need to include generic representative models with high accuracy and transient effects.

Battery models can be classified according to their modeling areas as given in [2]. They are mainly classified as Electrochemical, Mathematical, Electrical and Polynomial. Electrochemical models use the fundamental battery chemistry while mathematical models use empirical equations and polynomial models to study their responses in terms of polynomial expressions. Electrical models employ the passive circuit elements like capacitors, inductors and resistors to model electrolyte, electrode, polarization resistances along with active current and voltage sources. Literature review recommends that the circuit-based models are ideally suitable for thermal studies. An equivalent electrical circuit model approach has been used in this paper to develop the new battery model as it can be directly applicable to connect with other dynamic models like hybrid energy systems and electric vehicle modeling. Internal resistance of any battery model along with the thermal effect is also important to get accurate results. Thermal (temperature) effect is useful up to a certain level to carry the electrons from one electrode to the other through the electrolyte while increasing the rate of reaction. Once the saturation temperature is reached temperature effect beyond that level could be harmful to the battery life [3].

The effect of temperature is included explicitly in this paper along with all other variables in the new dynamic battery model. The model presented here is being validated finally with the comparison of simulation results and with known experimental data. The simulation results show that the model can produce more accurate and repeatable results than the existing models. This feature is discussed later in the paper with the comparison of known experimental results. The model was developed by considering the battery open circuit voltage change due to discharging and charging currents. The other key feature of the model is that it could be used to model any type of

Eng. S M Wijewardana, B.Sc.Eng(Mech) (Moratuwa), M.Eng(EEE)(Victoria), PGDip.Digital Control(Victoria), PGDipEd (Victoria), C.Eng, MIE(Sri Lanka), PhD Student, SEMS, Queen Mary University of London,

Email: s.m.wijewardana@qmul.ac.uk 
batteries like Lithium-ion, Lead-acid, Nickelcadmium, or Nickel-Metal-Hydride(NIMH). The other contributions made in this paper are the new table of battery parameters which reduces the modeling error within $2 \%$ of the known experimental results. Model can be used explicitly to test the fast charging/discharging processes of actual batteries with high currents. Also, the new model is capable of simulating a wide range of battery terminal voltage variations than the existing models that can accommodate around 1 to 1.5 volt change when charging/discharging takes place. In addition to the above contributions in this paper, a modified improved dynamic battery model is presented for the model given by Saiju et al [5], which can be used to simulate for a wide range of battery terminal output variations.

This paper is organized as follows. Section 2 describes the dynamic modeling of battery types and their associated physics with the governing mathematical relationships. Temperature effect on battery parameters have been discussed in section 3 . The new dynamic battery model is presented in section 4 along with the solutions to the prevailing questions raised by many researchers. Known experimental results have been used to test and validate the new model in section 5 . In table- 1 presents the accurate improved battery parameters for the generic equation which lead to reduce the percentage error less than $2 \%$ compared with the existing battery models. Conclusion is given in section 6 .

\section{Dynamic Modeling of Lithium- ion, Lead-acid, NiMH Batteries}

An accurate mathematical model is a starting point for a development of any superior dynamic battery model. Modeling approaches to Lithium-ion, Lead-acid or NiMH battery systems are quite similar with minor changes to the governing equations that represent the chemistry of each type. During the recent past Lithium-ion batteries have become very popular due to promising advantages and successful results when used in hybrid electric vehicles (HEV). Their high nominal voltage, high energy density, long life and none memory effects were the other reasons for preferred applications in HEV. Though, the new battery model presented here was developed by using Matlab/Simulink and related mathematics for Lithium-ion batteries, the complete model given at the end included the necessary changes to accommodate other battery models like Lead-acid, NiMH etc.

The simplest, generic battery model which was presented by MathWorks Inc. [4], is simulated here using Simulink.

$V_{\text {Bat }}$ : Nonlinear battery terminal voltage $(\mathrm{V})$

$E_{0}$ :Constant or open circuit voltage (V)

$\operatorname{Exp}(\mathrm{s}):$ Exponential zone dynamics (V)

Sel(s): Represents the battery mode. $\operatorname{Sel}(\mathrm{s})=0$

during battery discharge, $\operatorname{Sel}(\mathrm{s})=1$ during battery charging.

$K$ : Polarization constant $\left(\mathrm{Ah}^{-1}\right)$ or polarization resistance (Ohms)

$i^{*}$ : Low frequency current dynamics (A)

$i$ : Battery current (A)

it : Extracted battery capacity (or q) (Ah)

$Q$ : Maximum battery capacity (Ah)

$A$ : Exponential voltage $(\mathrm{V})$

$B$ : Exponential capacity $(\mathrm{Ah})^{-1}$

For aLead-acid battery the governing equations for charging/discharging are given by:

For discharging [4]: $\left(i^{*}>0\right)$ :

$$
f_{1}(i t, i *, i, \operatorname{Exp})=E_{0}-K \cdot \frac{Q}{Q-i t} . i *-K \cdot \frac{Q}{Q-i t} . i t+\text { Laplace }^{-1}\left(\frac{\operatorname{Exp}(s)}{\operatorname{Sel}(s)} \cdot 0\right)
$$

For charging [3]: $\left(i^{*}<0\right)$ :

$$
f_{2}(i t, i *, i, \operatorname{Exp})=E_{0}-K \cdot \frac{Q}{i t+0.1 \cdot Q} i *-K \cdot \frac{Q}{Q-i t} i t+\operatorname{Laplace}^{-1}\left(\frac{\operatorname{Exp}(s)}{\operatorname{Sel}(s)} \cdot \frac{1}{S}\right)
$$

For a Lithium-ion battery the governing equations as given in [4] were presented for reference:

For discharging $(i$

$*>0)$ :

$$
f_{1}(i t, i *, i)=E_{0}-K \cdot \frac{Q}{Q-i t} . i *-K \cdot \frac{Q}{Q-i t} . i t+A \cdot \exp (-B . i)
$$

For charging $\left(i^{*}<0\right)$ :

$f_{2}(i t, i *, i)=E_{0}-K \cdot \frac{Q}{i t+0.1 \cdot Q} . i *-K \cdot \frac{Q}{Q-i t} . i t+A \cdot \exp (-B . i t)$

For Nickel-cadmium and NiMH [4]:

For discharging $\left(\mathrm{i}^{*}>0\right)$ :

$$
f_{1}(i t, i *, i, \operatorname{Exp})=E_{0}-K \cdot \frac{Q}{Q-i t}, i *-K \cdot \frac{Q}{Q-i t} . i t+\text { Laplace }^{-1}\left(\frac{\operatorname{Exp}(s)}{\operatorname{Sel}(s)} \cdot \frac{1}{s}\right)
$$

For charging $\left(\mathrm{i}^{*}<0\right)$ :

$f_{2}(i t, i *, i, \operatorname{Exp})=E_{0}-K \cdot \frac{Q}{|i t|+0.1 \cdot Q}, i *-K \cdot \frac{Q}{Q-i t} . i t+\operatorname{Laplace}^{-1}\left(\frac{\operatorname{Exp}(s)}{\operatorname{Sel}(s)} \cdot \frac{1}{s}\right)$

The Lithium-ion battery model which was given by MathWorks Inc. [4] can be simulated as given by Fig.1. Dynamic Lead-Acid battery model which was presented by Saiju, R. et alin [5] was revisited and simulated here for analysis. The model defined in [5] was specially selected for comparison as it was based on the specific gravity (SG) of the battery acid to 
calculate the battery terminal voltage and the open circuit voltage $\left(V_{O C}\right)$. Also it was noted that the model defined in [5] has not included any temperature effect and the change of $V_{O C}$ due to $S O C$

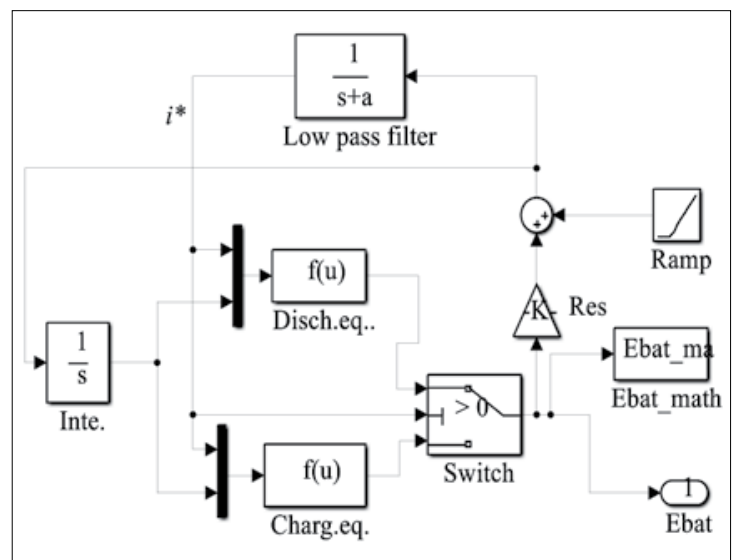

Figure1 - Battery model given by MathWorks Inc Ltd [4].

As the $S G$ changes nonlinearly in every fraction of a second when discharging, unlike in the case of the open circuit voltage $V_{O C}$, or in the case of the current measurements, practical difficulties could arise in the implementation of the dynamic model. However, compared to impedance spectroscopy test or hybrid pulsepower capability (HPPC) and the current impulse tests utilized by many manufacturers/researchers in $[1,7,8,10]$, the $S G$ test and the direct current measurement from the battery are cost-effective $[1,8]$ and may be more visual than expensive HPPC and the current impulse tests.

As described before, from the simulation results in [5] it was noted that the model can handle only 1volt change of the battery terminal voltage. It was modelled by using the equation (7) given below:

$V_{O C}=0.84+S G$.

When examined carefully, the equation (7) does not seem to give the correct results based on a $S G$ value of 1.2 for the battery acid. The result obtained for the maximum $V_{O C}$ would be $2.04 \mathrm{~V}$ according to (7) which is unrealistic. It was noted that simulation results used a $12 \mathrm{~V}$ battery with a capacity of 90Ah. Therefore, more appropriate equation based from reference, Ross MMD [6]is used to calculate the $V_{O C}$. Thus the $V_{O C}$ is assumed to be given by equation (8),[6]:

$V_{O C}=-168.22968+174.1360 \times S G-1.4836919 \exp (S G)$

$-169.0027 \times S G^{1 / 2} \times \log (S G)-0.00077765 / \log (S G)$
The specific gravity of the battery acid is an implicit function of the gassing effect $i_{\text {gas }}$.

Details can be referred in [6].

$$
S G=S G_{f u l l}-A h D\left(\frac{S G_{\text {full }}-S G_{\text {empty }}}{D K}\right)
$$

$S G$ is the specific gravity of the acid and the $S G_{\text {full }}$ and $S G_{\text {empty }}$ are the specific gravities when the battery is fully charged and when the battery is fully discharged respectively. $A h D$ is the number of $A h$ that would have to be discharged from a fully charged battery to bring it to the current state. The parameter $I$ is the normalised charge current and $I$ is negative when discharging. The Simulink block diagram for the dynamic model presented in [5] is shown by Fig.2. The generic battery model presented by MathWorks Inc [4] has not taken into account of temperature and gassing effect (for Li-Ion batteries the effect can be considered as minimal for certain applications)

I_bat : Battery current

I_gas : Gassing effect

$V \_b a t:$ Battery terminal voltage

SOC : State of charge

$A h D$ : Ampere.hour discharged

$A h_{\text {nom: }}$ Nominal ampere hour of the battery.

$D K$ : Fit parameter corresponding to the discharge capacity of reference cell at an infinitesimal discharge current

Ah : Ampere.hour

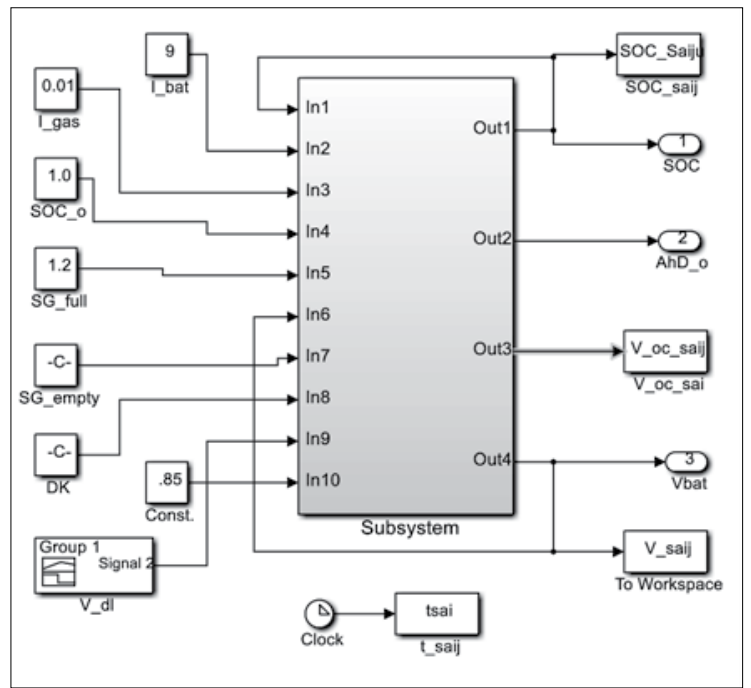

Figure 2- Simulink block diagram for Saijuet al [5].

The block diagram presented by Fig.2. was used the empirical equation (7). This model is improved and modified by the author to yield correct simulation results by using equation (8). The dynamic battery model presented by Saiju et al [5] can be validated with the improved modified model presented by the author as 
given by Fig.3. Due to ongoing research work many experimental parameters from [5] were not available for simulations. The temperature effect was taken into account when calculating the $D K$ fit parameter and the gassing effect.

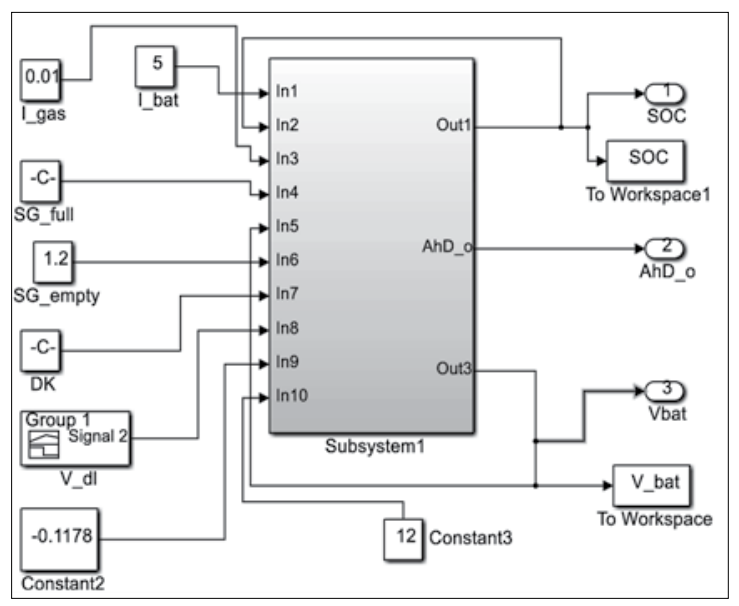

Figure 3 - Modified improved battery model for Saiju et al [5]

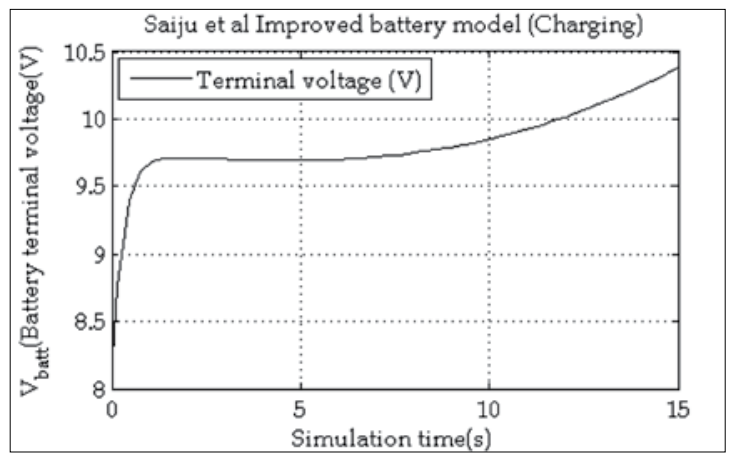

Figure 4- Simulation results of Saiju et al [5] improved model.

\section{Temperature Effect on Batteries}

The temperature makes a difference on three battery parameters of an equivalent electrical circuit battery model. They are the polarization voltage $K$, battery constant $E_{0}$ and exponential coefficients $A$ \& $B$ (eq.10). The polarization voltage represents the change of cell voltage from its open-circuit voltage when charging and discharging takes place. To make the model simpler, the thermal effect on $A$ has been ignored since $A \& B$ are highly related [7]. The coefficient $B$ takes a significant difference on the exponential part of the characteristic curve as given in [7]. The mathematical relationships as given in [7]:

$$
\begin{aligned}
& x_{n}=f(T)=A+B T+C T^{2} \\
& n=f\left(K, E_{0}, B, C\right)
\end{aligned}
$$

The equation for the battery terminal voltage, Eas given in [7]:
$E(q, T)=E_{0}-i R_{\text {int }}$

Where:

$R_{\text {ints: }}$ Battery internal series resistance

$T$ :Ambient temperature of the battery cell

$E_{0}$ : Battery constant

$$
\begin{gathered}
x_{k}(T)=f(T)=0.876-0.028 T+4.218 \times 10^{-4} T^{2} \\
x_{B}(T)=f(T)=0.733-0.045 T+9.63 \times 10^{-4} T^{-2} \\
x_{E 0}(T)=f(T)=0.986+4.97 \times 10^{-4} T-6.6 * 10^{-5} T \\
E(q, T)=E_{0}-K^{*}\left(\frac{Q}{Q-q}\right)+A \exp ^{-B q}
\end{gathered}
$$

Combining equations (10),(11),(12),(13),(14),(15) and (16) a generalized equation is presented as in Kroeze R et al [8]:

$$
E(q, T)=x_{E 0}(T) \times E_{0}-x_{K}(T) \times K\left(\frac{Q}{Q-q}\right)+A \exp ^{-x_{B} B q}
$$

Where:

$E(q, T)$ : No load voltage $(\mathrm{V})$

$E_{0}$ : Battery constant voltage $(\mathrm{V})$

$K$ : Polarization voltage(V)

$Q$ : Battery capacity(Ah)

$A, B$ : Exponential constants

$q$ : Charge or extracted capacity(Ah)

Simulation data:

$K_{\text {ref }}=0.05$ (Polarization voltage)

$Q=0.830$ (Ah) (Battery capacity)

$E_{0 \_ \text {ref }}=3.55$ Volts; $A_{\text {ref }}=0.35$ (Exponential Coeff.)

$B_{\text {ref }}=0.18$ (Exponential Coeff.)

Dynamic model developed using equation (17) for the Tan et al [9] model is given by Fig. 5 .

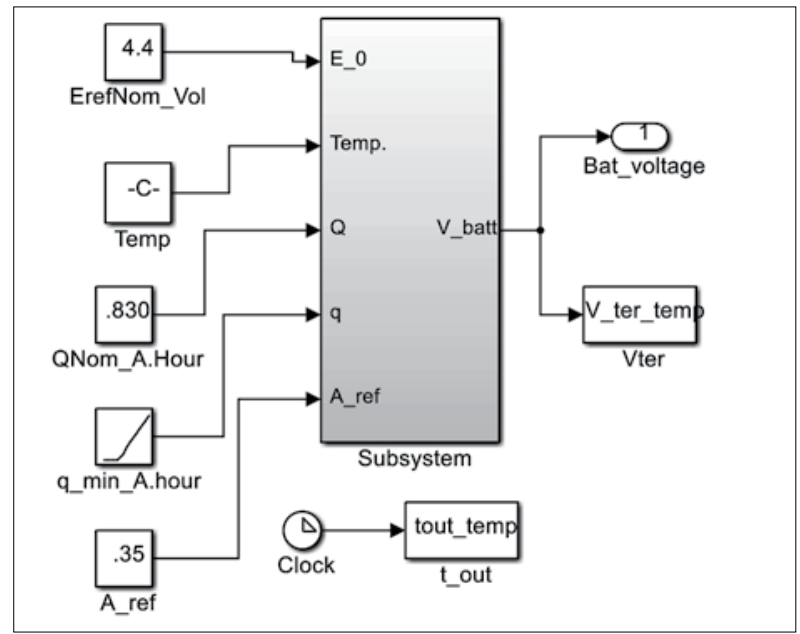

Figure 5 - Dynamic battery model for the Tan et al [9] with the temperature effect 


\section{New Dynamic Battery Model}

The author's model presented here is a, modified new improved dynamic battery model taking into consideration of the electrolyte temperature, cyclic charging and discharging, and the voltage stabilization effects. In this new model the battery terminal voltage variation is calculated by taking into account of transient and steady state behaviour of battery internal resistance with respect to SOC. The new modified model is a combination of the following battery models: Erdinc et al [7], Kroeze $\mathrm{R}$ et al [8], Tan et al [9] and the Chen et al [10] model. The key feature of this model is that:it can be applied to all types of Li-ion, Lead-acid and Nickel Metal Hydride (NiMH) batteries.

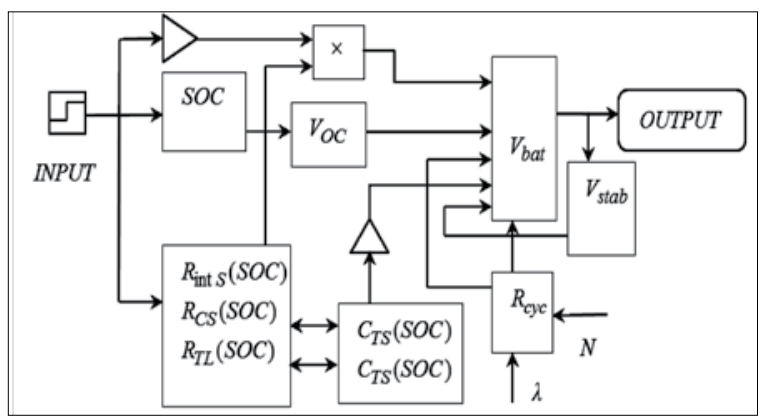

Figure 6 - Block diagram of the new battery
model

For electric vehicle and hybrid energy systems modeling, various equivalent electrical circuit models such as Shepherd's model, the RC model and the Thevenin model are very widely used $[13,19]$. An improved Thevenin's model which is also named as dual polarization (DP) model in literature [15] has been used here as it can be used to model any types of battery mentioned above. By comparison, the DP model has the best dynamic performance and provides the most accurate state of charge (SOC) estimation $[13,15]$. In addition to the DP model discussed above, the circuit diagram given by Fig.7. represents the improved Thevenin's equivalent electrical circuit which includes an additional loop to simulate the selfdischarging phenomenon due to prolonged storage of battery systems. The circuit parameter $R_{\text {self }}(T, n, S O C)$ signifies the resistance due to self-discharging and as it has a very high impedance and therefore the current passes through $R_{\text {selfis }}$ negligible [18] when normal discharge current is applied. However, $R_{\text {self }}$ highly depends on the long storage time, ambient temperature, SOC, and $n$ (when $n$ is a very large number). The parameter $n$ (orN) signifies the number of cycles the battery charging/discharging takes place. Combined impedance of $R_{\text {ints, }}, R_{\text {cyc }}$ in series with parallel $R C$ loops as shown by Fig. 7 is sometimes called the Warburg impedance [14].

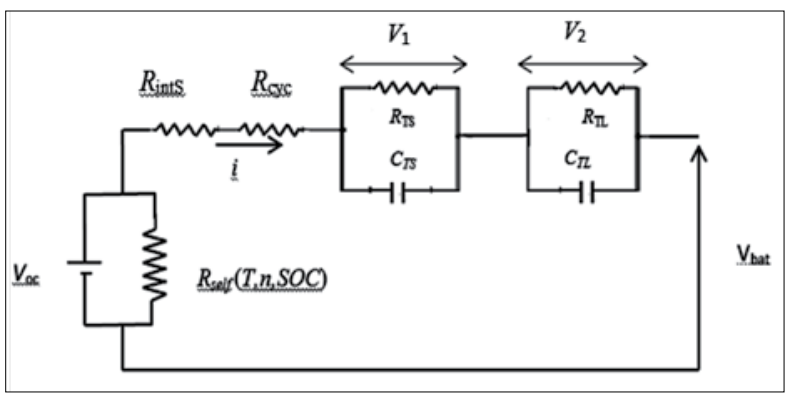

Figure 7 - Equivalent electrical circuit model

The variable equivalent internal impedance of the battery which is equal to the battery equivalent internal impedance consists of a series resistors composed of $R_{\text {int } S}$ and $R_{c y c}$, and two RC networks composed of $R_{T S}, C_{T S}$, $R_{T L}$ and $C_{T L}$. The battery equivalent internal impedance $\left(R_{\mathrm{int}} S\right)$ contributes to the instantaneous drops in the battery terminal voltage as given in [7]. The passive circuit elements $R_{T S}$ and the $C_{T S}$ shown in Fig.7, represent the fast dynamics associated with the reaction kinetics between the electrical charges at the electrode's surface barrier and the charges in the electrolyte. The $R_{T S}$ is the charge transfer resistance and the capacitance $C_{T S}$ models the electrochemical double layer capacitance. The $R_{T L}$ and the $C_{T L}$ circuit elements represent the slower dynamics of the cell in the order of hours. They are more representative of the battery chemistry related to the diffusion processes in the electrolyte and active electrode material. All these parameters are functions of the ambient temperature, electrolyte temperature, SOC, and the battery current. The components of $R C$ networks (two loops as shown in Fig. 7) are responsible for the short and the long-time transients in the battery internal impedance. Ideally this equivalent circuit should include two additional parallel loops to account for hysteresis phenomena of the battery. When the additional loops are included, both the short hysteresis and the long hysteresis behavior [13] of the battery can be predicted. The hysteresis phenomenon is caused due to parasitic behavior of the capacitors and is considered to be minimal and 
therefore it is being ignored in this model but could be included if and when necessary. From Fig.7., by using the Kirchhoff's law, the equations (18) and (19) could be derived.

$$
\begin{aligned}
\frac{d V_{1}}{d t}= & \frac{i}{C_{T S}}-\frac{V_{1}}{R_{T S} C_{T S}} . \\
\frac{d V_{2}}{d t}= & \frac{i}{C_{T L}}-\frac{V_{2}}{R_{T L} C_{T L}} . \\
V_{b a t}= & V_{o c}(S O C, T)+i\left(R_{\mathrm{int} s}+R_{c y c}\right)+V_{1}+V_{2}+\beta V_{s t a b} \\
& \pm T\left(C_{T}+C_{G}\right) e^{-\frac{\rho}{t}}
\end{aligned}
$$

The effect of $R_{\text {int } S}, R_{T S}, C_{T S}$, and $C_{T L}$ due to the battery $S O C$ can be calculated as in [7]. The improved new modified generic equation is presented here with as shown by equation (21):

$R_{c g}(S O C)=C_{g 1} \times \exp \left(-C_{g 2} \times S O C\right)$

$+C_{g 3} \times \exp \left(C_{g 4} \times S O C\right)$.

The constants $C_{g 1}, C_{g 2}, C_{g 3}$ and $C_{g 4}$ are

presented in the table 1 . below:

\section{Table. 1 - Improved new}

constants $C_{g 1}, C_{g 2}, C_{g 3}$ and $C_{g 4}$ in equation 21.

\begin{tabular}{|l|l|l|l|l|}
\hline$R_{c g}(S O C)$ & $C_{g 1}$ & $C_{g 2}$ & $C_{g 3}$ & $C_{g 4}$ \\
\hline$R_{\text {int } S}(S O C)$ & 1.21 & -52.1 & 0.0435 & 0.00941 \\
\hline$R_{T S}(S O C)$ & 0.1562 & -24.37 & 0.04669 & \\
\hline$C_{T S}(S O C)$ & 1100 & .1500 & -1200 & -7.100 \\
\hline$R_{T L}(S O C)$ & 1.321 & -34.72 & 0.0491 & 0.0071 \\
\hline$C_{T L}(S O C)$ & 5200 & - & -8350 & -19.4 \\
\hline
\end{tabular}

Battery internal resistance change due to cyclic effect is assumed to be given by the empirical equation (22), as in [7]:

$$
R_{c y c}=\xi \times n^{\lambda} \text {. }
$$

In equation (22), nis the discharge/charge cycle number and $\xi$ is a constant. The constant $\lambda$ is normally taken to be equal to $1 / 2$ according to [7]. The modified governing equation used for the development of the new model is given by equation (20). As the battery terminal voltage is a function of SOC and the battery temperature, battery model given by Fig.6. is further modified to include the ambient temperature and the terminal voltage stabilization effects (Fig. 13). The Model differs from the other models as the $C_{\mathrm{TS}}$ and the $C_{\mathrm{TL}}$ effect that will create a transient effect on the internal resistance of the battery which causes the capacitive feedback effect. This causes the terminal voltage discharge deceleration along with the SOC. All existing models discussed above used the battery current $I_{b a t}$ along with the impedances to model the Battery terminal voltage. In this model $\operatorname{Voc}(S O C)$ which will vary according to the $S O C$ has been taken into account(more accurate than using current) to calculate the feedback effect of the terminal battery voltage than $I_{\text {bat. }}$ In equation (20) the parameters $C_{T}, C_{G}$ and $\rho$ are constants to generalise the electrolyte temperature effect due to electron transfer which causes the nonlinear behaviour of rate of reaction and the ionization of electrolyte. The battery open circuit voltage $V o c$ is defined as the difference of the electrical potential between the two terminals of a battery, when there is no external load connected [7]. As the value of battery open circuit voltage is strongly dependent on battery $S O C$, it can be calculated (equation 23) as given in [7].

$\operatorname{Voc}(S O C)=-1.031 \times \exp (-35 \times S O C)+3.685$

$+0.2156 \times S O C-0.1178 \times S^{S O C^{2}}$

$+0.321 \times S^{2} C^{3}$

The battery SOC can be expressed as given in [2],[19]:

$S O C=S O C_{\text {init }}-\int \frac{i_{\text {bat }}}{C_{\text {usable }}} * d t$

Where:

$I_{b a t}$ : Battery current (A)

SOC: State of charge

$S O C_{\text {init }}$ : Initial state of charge

$C_{\text {usable }}:$ Usable battery capacity $(\mathrm{Ah})$

$T$ : Electrolyte temperature $\left({ }^{\circ} \mathrm{C}\right)$

$t$ : Time (seconds)

\section{Simulation Results of the Improved New Dynamic Model}

In this section and in the conclusion mainly, the new model simulation results will be compared Companson of models with the New Battery Model

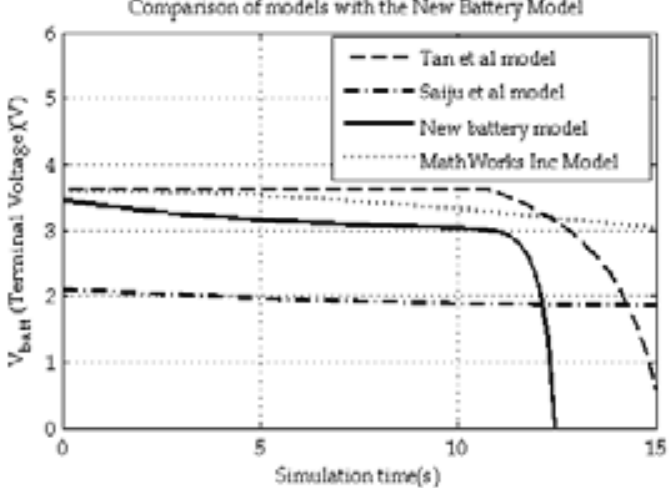

Figure 8 - Comparison of the new model simulation results with the existing models with fast-discharging 
with the simulation results from existing battery models and known experimental data from Tan et al [9] and the Chen et al [10] experiments.

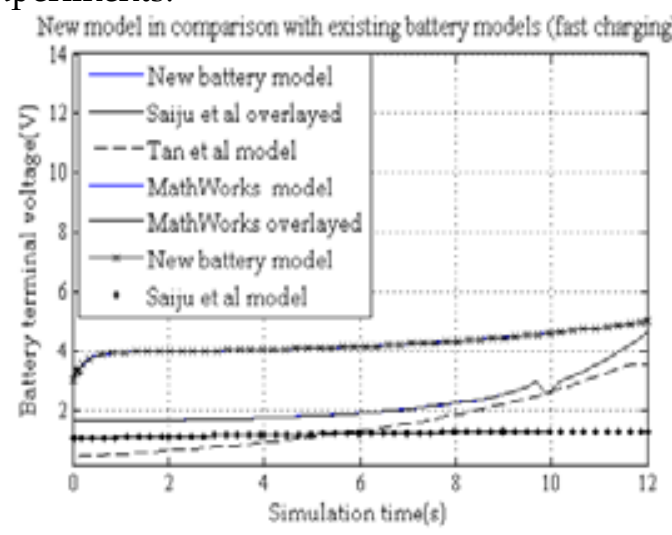

Figure 9 - Comparison of the new model simulation results with the existing model

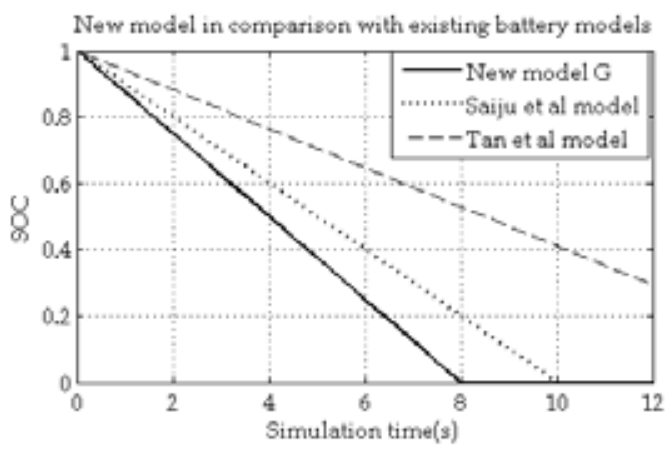

Figure 10 - Comparison of the new model simulation results with the existing models

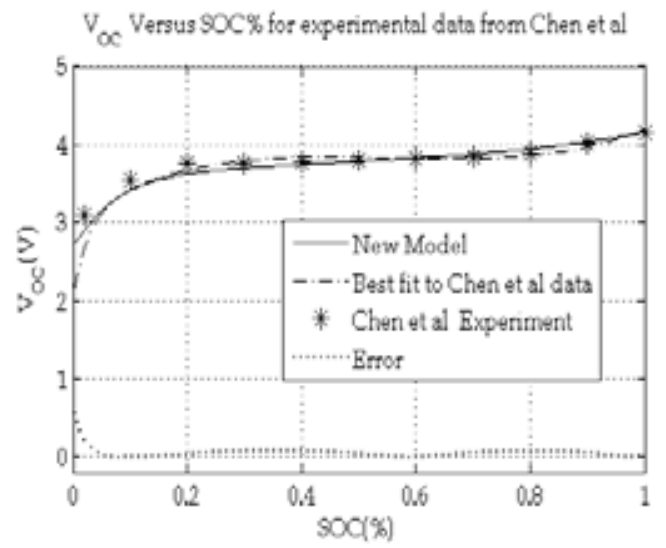

Figure 11 - Comparison of the new model simulation results with known experimental results from Chen's et al [10] experiment.

The new parameter values as given by table 1 was generated by using powerful Simulink tool boxes and is another by product to validate the new model and can be used to model future battery models. In addition, by comparing Fig. 4 and 9 modified improved battery model presented for Saiju et al [5] can be validated as the simulation results are very successful.

\subsection{Extension of the New Model to Include the Ambient Temperature Effect.}

The model presented by Fig.6 is further developed to include the ambient temperature fluctuations. The eq. (20) given earlier defines the electrolyte temperature change due to electron transfer between the anode and the cathode. This equation is further modified to include the ambient temperature effect and will be discussed in this section in detail.

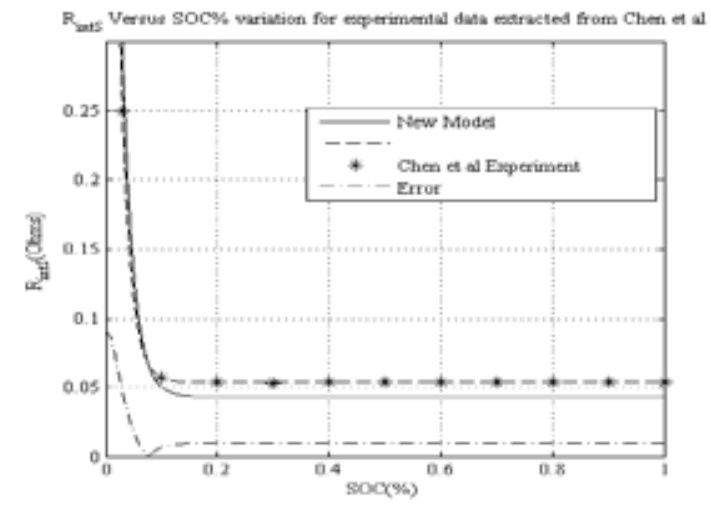

Figure 12 - Comparison of the new model simulation results with known experimental results from Chen's et al [10] experiment

The available battery capacity $C_{\text {usable varies }}$ depending on the capacity fading. The effect of capacity fading and other cyclic resistances were included in the extended new model and the constants were evaluated when tuning. The values of $R_{\text {ints, }} R_{\mathrm{TS}}, C_{\mathrm{TS}}, R_{\mathrm{TL}}$ and $C_{\mathrm{TL}}$ due to battery SOC can be calculated as given below [7]:

$$
\begin{array}{r}
R_{\text {ints }}(S O C)=0.1562 \times \exp (-24.37 \times S O C)+0.07446 \\
\ldots .(25) \\
R_{\mathrm{TS}}(S O C)=0.3208 \times \exp (-29.14 \times S O C)+0.04669 . \\
\ldots .(26) \\
C_{\mathrm{TS}}(S O C)=752.9 \times \exp (-13.51 \times S O C)+703.6 . \\
\ldots .(27) \\
R_{\mathrm{TL}}(S O C)=6.603 \times \exp (-155.2 \times S O C)+0.04984 . \\
\ldots .(28) \\
C_{\mathrm{TL}}(S O C)=-6056 \times \exp (-27.12 \times S O C)+475 .
\end{array}
$$

In addition to the equations (18), (19), (20), (21), (22) (23) and (24), the ambient temperature effect of the model is defined by the equations (30), (31), (32),(33) and (34) given below:

$$
\begin{aligned}
& V_{O C}(q, T)=x_{V_{O C}}(T) V_{O C}(0,0) \\
& +x_{k}(T) K\left(\frac{Q}{Q-q}\right)+A \exp ^{-x_{B} B q} . \\
& x_{V_{O C}}(T)=0.986+4.97 \times 10^{-4} T-6.6 \times 10^{-5} T^{2} .
\end{aligned}
$$




$$
\begin{aligned}
& x_{k}(T)=0.876-0.028 T+4.218 \times 10^{-4} T^{2} \ldots \\
& x_{B}=0.733-0.055 T+9.63 \times 10^{-4} T^{2} .
\end{aligned}
$$

The constants $A$ and $B$ are the constants associated with the exponential term in equation (30). The symbol $Q$ is the total battery capacity in $A h$ and the $q$ is the available battery capacity which is normally symbolizes as $C_{\text {usable, }}$ at any instant. $V_{O C}$ is the battery open circuit voltage at the start. The equation (20) given earlier is now modified to include the ambient temperature effect and is given by equation (34):

$$
\begin{aligned}
& V_{\text {bat }}=i\left(R_{\text {int } s}+R_{c y c}\right)+V_{1}+V_{2} \pm V_{O C}(q, T) \\
& +\beta V_{\text {stab }}+T\left(C_{T}+C_{G}\right) e^{-\rho / t} .
\end{aligned}
$$

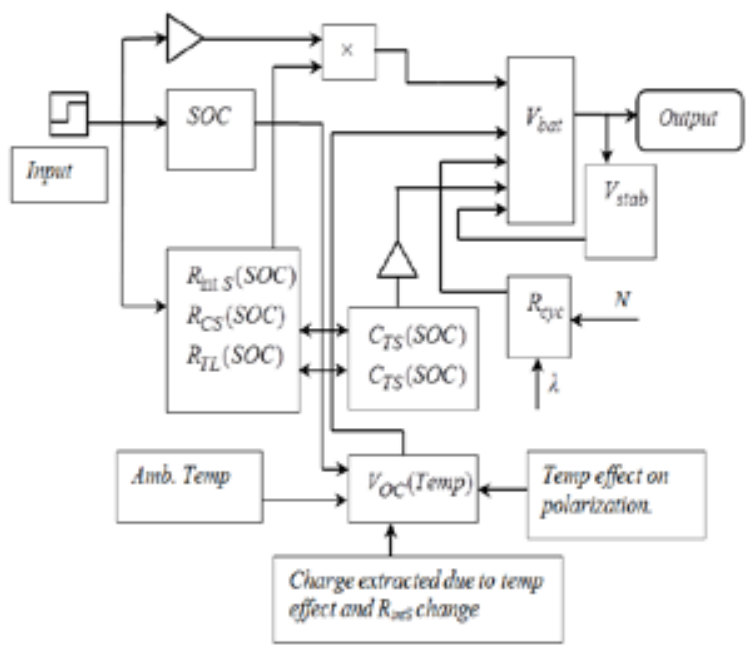

Figure 13 - Block diagram of the new model extended to include the ambient temperature effect.

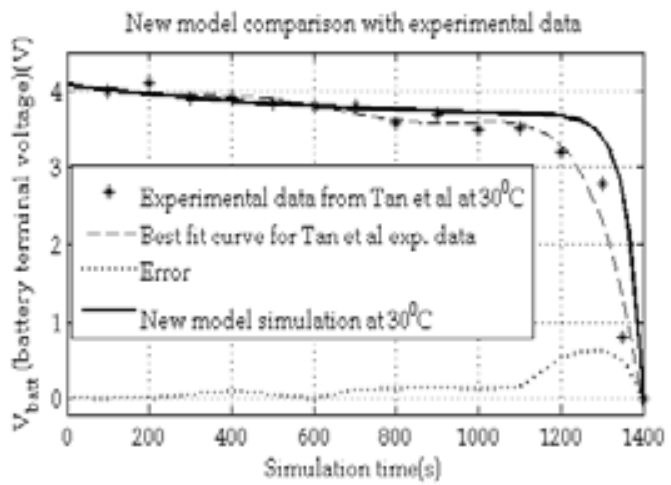

Figure 14 -Temperature effect of the new model in comparison with the Tan et al [9] exp. data

The new model represented by Fig. 13 includes the ambient temperature effect in addition to the internal electrolyte temperature change due to electron diffusion.
The constants used in the dynamic model were selected carefully within the constraints of the manufacturers' parameters. Battery internal resistance cannot increase beyond $0.1 \mathrm{Ohms}$. CGR18650A cylindrical Li-ion battery specifications were considered to study input data where necessary. Maximum value for SOC is 1 and the minimum value can be zero or 0.1 [19]. The model can be used to model hybrid vehicle battery systems of Li-ion, Lead acid or NiMH batteries. The simulation results were very good and the proposed new model can be identified as an accurate new model to apply for dynamic modeling.

\section{Conclusion}

Simulation results from Fig.11,12 and Fig. 13 confirm the model accuracy and the average error is within $2 \%$ which is a successful achievement in modeling. With the known experimental results from Tan et al [9] and Chen et al [10] experiments, the new model simulation results can be validated. Extension of the new model which was given by Fig.13 that includes the ambient temperature effect was validated with the comparison of experimental results from Tan's et al [9]. This simulation results and the experimental data are shown by Fig. 14. From the simulation results it was observed that the battery cell temperature cannot increase more than $38^{\circ} \mathrm{C}$ and the ambient temperature can decrease as low as $-10^{\circ} \mathrm{C}$ for which the model proved to be working successful. When the temperature increases more than $38^{\circ} \mathrm{C}$ the behavior of the battery terminal voltage is unusual [20] as expected and therefore it is necessary to deploy a proper cooling system to avoid further increase in the electrolyte temperature. The simulation results show that the model can accommodate a wider range of battery terminal voltages than the existing models. The new battery model presented here could provide satisfactory results and offer good solutions for many questions that simulation engineers face with. It is currently being used to develop reliable hybrid energy power systems. Thus the model developed in this paper are proving to be extremely useful for both simulation and estimation studies. 


\section{References}

[1] Smith, A. K., "Electrochemical modeling, estimation and control of lithium-ion batteries, "PhD Thesis in Mechanical Engineering The Pennsylvania State University. The Graduate School Dec. 2006.

[2] Zoroofi, S., "Modeling \& simulation of vehicular power systems." M.Sc. Thesis, Dept. of Energy and Environment, Division of Power Engineering Chalmers University of Technology Goteborg Sweden, 2008.

[3] Zhang, T., "The Economic Benefits of Battery Energy Storage System in Electric Distribution System" M.Sc. Thesis, Electrical and Computer Engineering, Worcester Polytechnic Institute, 2013.

[4] MathWorks Inc., "Implement Generic Battery Model" Available Online: http://www.mathw-

orks.co.uk / webhp/Accessed on, 16 Dec 2013

[5] Saiju, R., Heier, S., “ Performance Analysis of Lead Acid Battery Model for Hybrid Power System", IEEE 2008.

[6] Ross, M. M. D., "A simple but comprehensive Lead- Acid battery model for Hybrid System Simulation" 1471 Boul, Lionel- Bouled, Suite 26, Varennes, Quebec J3X 1P7. Available online:Article from www. RERinfo.ca, accessed on 18 Dec 2013.

[7] Erdinc, O., Vural, B., Uzunoglu, M., “A dynamic lithium-ion battery model considering the effects of temperature and capacity Fading" IEEE, 2009.

[8] Kroeze, R. C., Krein, P. T., “Electrical Battery Model for Use in Dynamic Electric Vehicle Simulations, University of Illinois at Urbana- Champaign Depart. of Electrical and Computer Engineering 1406 W. Green St. Urbana, IL 61801. IEEE 2008.

[9] Tan, Y. K., Mao, J. C., \& Tseng, K. J., "Modelling of Battery Temperat. Effect on Electrical Characteristics of Li-ion Battery in Hybrid Electric Vehicle" IEEE PEDS 2011, Singapore, Dec 2011.

[10] Chen, M., Rincon-Mora, G. A., "Accurate Electrical Battery Model Capable of Predicting Runtime and I - V Performance IEEE transactions on energy conversion, vol. 21, no. 2, June 2006.
[11] Bhide, S., Shim, T., “Development of Improved Lithium- Ion Battery model incorporating thermal \& rate factor effects", University of Michigan USA, 978-1-4244-2601 IEEE, 2009.

[12] Knauff, M., McLaughlin, J., Dafis, C., et al. "Simulink Model of a Lithium-Ion Battery for the Hybrid Power System Testbed".

[13] Oliver, T., Dessaint, L- A., “Experimental Validation of a Battery Dynamic Model for EV Applications", Elect. Engineering Department, 'Ecole de Technologie Sup' erieure 1

Email: olivier.tremblay.1@ens.etsmtl.ca

[14] Vepa, R., “Dynamic Modeling, Simulation and Control of Energy Generation", 1 15Ted., Springer, London, 2013, 331p

[15] He, H., Xiong, R., and Fan, J., "Evaluation of Lithium-Ion Battery Equivalent Circuit Models for State of Charge Estimation by an Experimental Approach" ISSN 1996-1073 Open Access Energies 2011,

[16] Vairamohan, B., "State of charge estimation for batteries." M. Sc. Dept. Electrical Eng., Thesis University of Tennesse, Knoxville, Dec 2002.

[17] Gao, L., and Liu, S., "Dynamic Lithium - Ion Battery Model for System simulation", IEEE Transactions on components and packaging technologies, Vol. 25, no. 3, September 2002.

[18] Sarasua, A. E., Molina, M G., and Mercado P. E., "Dynamic Modelling of Advanced Battery Energy Storage System for Grid Tied AC Microgrid Applications., Chap.-12, Available on :www.intechopen.com/.../dynamic modelling- of-advanced-battery-energ..23Jan 2013 E-Book, Accessed on 08 Dec 2013.

[19] Jiang, Y., Zhang, C., Zhang, W., Shi, W., \& Liu, Q. (2013). “Modeling charge polarization voltage for large lithium - ion batteries in electric Vehicles." Journal of Industrial Engineering and Management, 6(2), pp 686697, June 2013

[20] Wijewardana, S., "Maximum Power Point Analysis Using Simulink/Matlab for a Hybrid Solar Photovoltaic / Battery Storage system", International Journal of Emerging Technology and Advanced Engineering, Volume 3, Issue 11,November 2013. 
